\title{
Cooperative Manipulation and Transportation with Aerial Robots
}

\author{
Nathan Michael, Jonathan Fink, and Vijay Kumar \\ University of Pennsylvania \\ Philadelphia, Pennsylvania \\ Email: \{nmichael, jonfink, kumar\}@grasp.upenn.edu
}

\begin{abstract}
In this paper we consider the problem of controlling multiple robots manipulating and transporting a payload in three dimensions via cables. We develop robot configurations that ensure static equilibrium of the payload at a desired pose while respecting constraints on the tension and provide analysis of payload stability for these configurations. We demonstrate our methods on a team of aerial robots via simulation and experimentation.
\end{abstract}

\section{INTRODUCTION}

Aerial transport of payloads by towed cables is common in emergency response, industrial, and military applications for object transport to environments inaccessible by other means. Examples of aerial towing range from emergency rescue missions where individuals are lifted from dangerous situations to the delivery of heavy equipment to the top of a tall building. Typically, aerial towing is accomplished via a single cable attached to a payload. However, only limited controllability of the payload is achievable with a single attachment point [1].

In this work we address the limitations of aerial towing by designing cooperative control laws for multiple aerial robots that enable manipulation of a payload in three dimensions. While we formulate the general conditions for system equilibrium at the desired pose for an arbitrary number of robots, we focus on a system of three aerial robots for discussions of workspace and payload stability. We show that despite the fact that such a system is underactuated and limited by unilateral tension constraints, we are able to manipulate a payload to a desired pose (position and orientation) in simulation and experimentation. We extend the analysis to motion planning by exploring the set of possible robot configurations for a given payload pose.

While examples of cooperative multi-robot planar towing exist in the literature, there are significant differences between interacting with an object on the ground and in the air. In [2], the authors consider the problem of cooperative towing with a team of ground robots, where under quasi-static assumptions there is a unique solution to the motion of the object given the robot motions. This approach is not directly extensible to three-dimensional manipulation where frictional ground forces are absent and gravity introduces dynamics into the problem.

The cooperative aerial towing problem is similar to the problem of controlling cable-actuated parallel manipulators in three dimensions, where in the former the payload pose

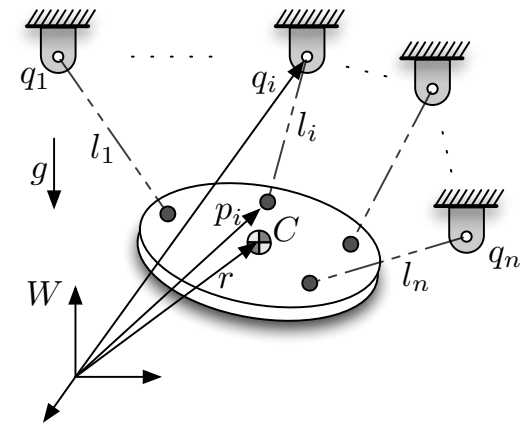

Fig. 1. A rigid body suspended by $n$ cables with world-frame pivot points $q_{i}$. Analysis techniques for cable-actuated parallel manipulators assume that $q_{i}$ is fixed while $l_{i}$ varies in magnitude, while for cooperative aerial manipulation we fix $l_{i}$ and vary $q_{i}$ by changing the positions of the aerial robots.

is affected by robot positions and in the latter pose control is accomplished by varying the lengths of multiple cable attachments (see Fig. 1). Thus the work on workspace analysis [3, 4], control [5], and static analysis [6] of such parallel manipulators is directly relevant to this paper.

More generally, we are interested in the mechanics of payloads suspended by $n$ cables in three dimensions. The $n=6$ case is addressed in the literature on cable-actuated platforms. When $n=5$, if the line vectors are linearly independent and the cables are taut, the line vectors and the gravity wrench axis must belong to the same linear complex [7]. The payload is free to instantaneously twist about the reciprocal screw axis. When $n=4$, under similar assumptions on linear independence and positive tension, the line vectors and the gravity wrench must belong to the same linear congruence. The unconstrained freedoms correspond (instantaneously) to a set of twists whose axes lie on a cylindroid. In the $n=3$ case, all three cables and the gravity wrench axis must lie on the same regulus - the generators of a hyperboloid which is a ruled surface [8]. Of course, in all of these cases there are special configurations in which the screw systems assume special forms [7] which are not discussed in this paper. The arguments for the $n=1$ and the $n=2$ cases are similar, but in these cases, the cables and the center of mass must lie on the same vertical plane for equilibrium.

We approach the development of controllers for cooperative aerial manipulation and transportation as follows: in Sect. II we formulate conditions for general static equilibrium of an 
object in three dimensions, after which we focus our discussion on systems with three robots. By deriving conditions of static equilibrium and analyzing these conditions based on bounded tension models and stability, in Sect. III we are able to identify valid system configurations for aerial manipulation that ensure that the payload achieves a desired pose. Through simplifying assumptions guided by our robot model, we are able to arrive at closed-form analytic solutions for the tensions in the cables and payload stability for a given system configuration. For the derivation of valid configurations we assume point-model robots. In Sect. IV, we develop and experimentally validate controllers for dynamic robots that respect this point-model and enable application of our methods to a team of quadrotors. We review and analyze simulation and experimental results in Sects. V and VI and conclude in Sect. VII.

\section{Problem Formulation}

\section{A. Mechanics of a cable-suspended payload}

We begin by considering the general problem with $n$ robots (quadrotors in our experimental implementation) in three dimensions. We consider point robots for the mathematical formulation and algorithmic development although the experimental implementation requires us to consider the full twelve-dimensional state-space of each quadrotor and a formal approach to realizing these point abstractions, which we provide in Sect. IV. Thus our configuration space is given by $\mathcal{Q}=\mathbb{R}^{3} \times \ldots \times \mathbb{R}^{3}$. Each robot is modeled by $q_{i} \in \mathbb{R}^{3}$ with coordinates $q_{i}=\left[x_{i}, y_{i}, z_{i}\right]^{\mathrm{T}}$ in an inertial frame, $W$ (Fig. 2). The $i^{\text {th }}$ robot cable with length $l_{i}$ is connected to the payload at the point $P_{i}$ with coordinates $p_{i}=\left[x_{i}^{p}, y_{i}^{p}, z_{i}^{p}\right]^{\mathrm{T}}$ in $W$. We require $P_{1}, P_{2}$, and $P_{3}$ to be non-collinear and span the center of mass. The payload has mass $m$ with the center of mass at $C$ with position vector $r=\left[x_{C}, y_{C}, z_{C}\right]^{\mathrm{T}}$. The payload's pose $\mathbf{A} \in S E(3)$ can be locally parameterized using the components of the vector $r$ and the Euler angles with six coordinates: $\left[x_{C}, y_{C}, z_{C}, \alpha, \beta, \gamma\right]^{\mathrm{T}}$. The homogeneous transformation matrix describing the pose of the payload is given by:

$$
\mathbf{A}=\left[\begin{array}{cc}
\mathbf{R}(\alpha, \beta, \gamma) & \left(\begin{array}{c}
x_{C} \\
y_{C} \\
z_{C}
\end{array}\right) \\
0 & 1
\end{array}\right] .
$$

Note that $\mathbf{R}$ is the rotation matrix going from the object frame $B$ to the world frame $W$ (as depicted in Fig. 2). Additionally, for this work we follow the Tait-Bryan Euler angle parameterization for $\{\alpha, \beta, \gamma\}$.

The equations of static equilibrium can be written as follows. The cables exert zero-pitch wrenches on the payload which take the following form after normalization:

$$
\mathbf{w}_{i}=\frac{1}{l_{i}}\left[\begin{array}{l}
q_{i}-p_{i} \\
p_{i} \times q_{i}
\end{array}\right] .
$$

The gravity wrench takes the form:

$$
\mathbf{g}=-m g\left[\begin{array}{c}
e_{3} \\
r \times e_{3}
\end{array}\right]
$$

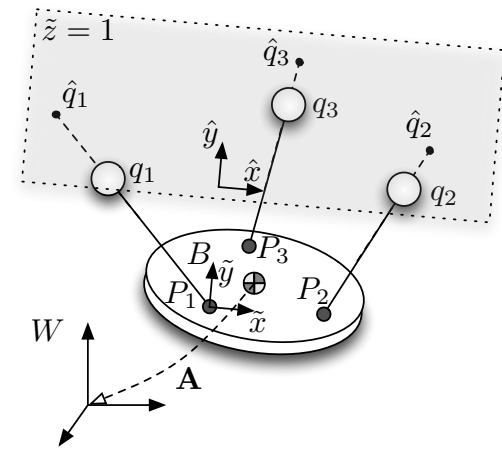

Fig. 2. A team of three point-model robots manipulate a payload in three dimensions. The coordinates of the robots in the inertial frame $W$ are $q_{i}=$ $\left[x_{i}, y_{i}, z_{i}\right]$ and in the body-fixed frame (attached to the payload) $B$ are $\tilde{q}_{i}=$ $\left[\tilde{x}_{i}, \tilde{y}_{i}, \tilde{z}_{i}\right]$. The rigid body transformation from $B$ to $W$ is $\mathbf{A} \in S E(3)$. Additionally, we denote the projection of the robot position $\tilde{q}_{i}$ along $q_{i}-p_{i}$ to the plane $\tilde{z}=1$ as $\hat{q}_{i}=\left[\hat{x}_{i}, \hat{y}_{i}, 1\right]$.

where $g$ is the acceleration due to gravity and $e_{3}=[0,0,1]^{\mathrm{T}}$. For static equilibrium:

$$
\left[\begin{array}{llll}
\mathbf{w}_{1} & \mathbf{w}_{2} & \cdots & \mathbf{w}_{n}
\end{array}\right]\left[\begin{array}{c}
\lambda_{1} \\
\lambda_{2} \\
\vdots \\
\lambda_{n}
\end{array}\right]=-\mathbf{g}
$$

where $\lambda_{i} \geq 0$ is the tension in the $i^{\text {th }}$ cable.

When $n=3$, in order for (2) to be satisfied (with or without non-zero tensions), the four line vectors or zero pitch wrenches, $\mathbf{w}_{1}, \mathbf{w}_{2}, \mathbf{w}_{3}$, and $\mathbf{g}$ must belong to the same regulus. The lines of a regulus are points on a 2-plane in $\mathbb{P R}^{5}[9]$, which implies that the body is underconstrained and has three degrees of freedom. Instantaneously, these degrees of freedom correspond to twists in the reciprocal screw system that are reciprocal to $\mathbf{w}_{1}, \mathbf{w}_{2}$, and $\mathbf{w}_{3}$. They include zero pitch twists (pure rotations) that lie along the axes of the complementary regulus (the set of lines each intersecting all of the lines in the original regulus). Geometrically, (2) simply requires the gravity wrench to be reciprocal to the reciprocal screw system, a fact that will be exploited in our calculations in the next section.

\section{B. Cooperative manipulation with three aerial robots}

From this point on, we will discuss the special case of a payload transported by three robots. The analysis for $n \neq 3$ is not very different. However the $n=3$ case is the smallest $n$ for which we can achieve equilibrium for a large set of specified three-dimensional poses of the payload ${ }^{1}$.

We will make the following simplifying assumptions for the $n=3$ case:

1) The payload is a homogeneous, planar object and the center of mass lies in the plane of the three pivot points.

\footnotetext{
${ }^{1}$ The $n=1$ case is a simple pendulum with only one stable equilibrium with a single orientation. The $n=2$ case limits our ability to achieve a desired angle of rotation about the line joining $P_{1}$ and $P_{2}$.
} 
2) The mass of the object is sufficiently small that three robots are able to lift the object.

3) The payload does not flip during manipulation, restricting the orientation to $|\alpha|<\frac{\pi}{2}$ and $|\beta|<\frac{\pi}{2}$.

4) We require the robots to assume positions that are on one side of the plane of the three pivot points.

For the analysis, we will use a local frame, $B$, attached to the payload that is defined with the origin at $P_{1}$, the $x$ axis pointing toward $P_{2}$ and the $x-y$ plane coincident with the plane formed by $P_{1}, P_{2}$, and $P_{3}$. In this local coordinate system, the components are denoted by $(\tilde{*})$ and given as:

$P_{1}=[0,0,0]^{\mathrm{T}}, \quad P_{2}=\left[\tilde{x}_{2}^{p}, 0,0\right]^{\mathrm{T}}, \quad P_{3}=\left[\tilde{x}_{3}^{p}, \tilde{y}_{3}^{p}, 0\right]^{\mathrm{T}} ;$ $\tilde{q}_{1}=\left[\tilde{x}_{1}, \tilde{y}_{1}, \tilde{z}_{1}\right]^{\mathrm{T}}, \quad \tilde{q}_{2}=\left[\tilde{x}_{2}, \tilde{y}_{2}, \tilde{z}_{2}\right]^{\mathrm{T}}, \quad \tilde{q}_{3}=\left[\tilde{x}_{3}, \tilde{y}_{3}, \tilde{z}_{3}\right]^{\mathrm{T}}$.

Note that without loss of generality, we assume that $\tilde{x}_{2}^{P}>\tilde{x}_{3}^{P}$, restricting the possible permutations of $P_{i}$. Equation (2) takes the form:

$$
\tilde{\mathbf{W}} \lambda=-\tilde{\mathbf{g}}
$$

with

$$
\begin{gathered}
\tilde{\mathbf{W}}=\left[\begin{array}{ccc}
\tilde{x}_{1} & \tilde{x}_{2}-\tilde{x}_{2}^{p} & \tilde{x}_{3}-\tilde{x}_{3}^{p} \\
\tilde{y}_{1} & \tilde{y}_{2} & \tilde{y}_{3}-\tilde{y}_{3}^{p} \\
\tilde{z}_{1} & \tilde{z}_{2} & \tilde{z}_{3} \\
0 & 0 & \tilde{y}_{3}^{p} \tilde{z}_{3} \\
0 & -\tilde{x}_{2}^{p} \tilde{z}_{2} & -\tilde{x}_{3}^{p} \tilde{z}_{3} \\
0 & \tilde{x}_{2}^{p} \tilde{y}_{2} & \tilde{x}_{3}^{p} \tilde{y}_{3}-\tilde{y}_{3}^{p} \tilde{x}_{3}
\end{array}\right] \\
\tilde{\mathbf{g}}=-m g\left[\begin{array}{c}
\mathbf{R}^{\mathrm{T}} e_{3} \\
{\left[\begin{array}{c}
\frac{1}{3}\left(\tilde{x}_{2}^{p}+\tilde{x}_{3}^{p}\right) \\
\frac{1}{3}\left(\tilde{y}_{3}^{p}\right) \\
0
\end{array}\right] \times \mathbf{R}^{\mathrm{T}} e_{3}}
\end{array}\right] .
\end{gathered}
$$

\section{MECHANICS OF 3-D MANIPULATION WITH CABLES}

\section{A. Robot positions for desired payload pose}

The first problem that must be solved is the analog to the inverse kinematics problem in parallel manipulators:

Problem 1 (Inverse Problem). Given the desired payload position and orientation (1), find positions of the robots, $q_{i}$, that satisfy the kinematics of the robots-cables-payload system and the equations of equilibrium (3).

The inverse kinematics problem is underconstrained. If the cables are in tension, we know that the following constraints must be true:

$$
\left(\tilde{x}_{i}-\tilde{x}_{i}^{p}\right)^{2}+\left(\tilde{y}_{i}-\tilde{y}_{i}^{p}\right)^{2}+\left(\tilde{z}_{i}-\tilde{z}_{i}^{p}\right)^{2}=l_{i}^{2},
$$

for $i=\{1,2,3\}$. We impose the three equations of static equilibrium (3) to further constrain the solutions to the inverse problem. This further reduces the degrees of freedom to three. Finally, we require that there exist a positive, $3 \times 1$ vector of multipliers, $\lambda$, that satisfies (3).

We solve this problem by finding the three screws (twists) that are reciprocal to the three zero pitch wrenches. Define the $6 \times 3$ matrix $\mathbf{S}$ of twists with three linearly independent twists such that the vectors belong to the null space of $\tilde{\mathbf{W}}^{\mathrm{T}}$ :

$$
\tilde{\mathbf{W}}^{\mathrm{T}} \mathbf{S}=0 \text {. }
$$

$\mathbf{S}\left(\tilde{x}_{i}, \tilde{y}_{i}, \tilde{z}_{i}\right)$ is an algebraic function of the positions of the quadrotors. In order to satisfy (3), $q_{i}$ must satisfy the three algebraic conditions:

$$
\mathbf{S}\left(\tilde{x}_{i}, \tilde{y}_{i}, \tilde{z}_{i}\right)^{\mathrm{T}} \tilde{\mathbf{g}}=0 .
$$

The inverse problem reduces to the problem of solving for the three-dimensional set $Q_{c} \subset \mathcal{Q}$ by solving for the nine variables $\left\{\left(\tilde{x}_{i}, \tilde{y}_{i}, \tilde{z}_{i}\right), i=1,2,3\right\}$ subject to $(4,5)$.

We now restrict our attention to a reduced space of possible configurations based upon the assumptions $(3,4)$. We introduce the notion of normalized components, denoted by $\left({ }^{\wedge}\right)$, with $\hat{q}_{i}=\left[\hat{x}_{i}, \hat{y}_{i}, 1\right]$ to define the position of the $i^{\text {th }}$ robot projected to a constant height $\tilde{z}_{i}=1$ above the payload. Based on this simplification (we now only need to solve for three planar positions), we redefine the static equilibrium condition as

$$
\mathbf{S}\left(\hat{x}_{i}, \hat{y}_{i}, 1\right)^{\mathrm{T}} \tilde{\mathbf{g}}=0
$$

Solving the system of equations in (6) yields algebraic solutions for $\left\{\hat{x}_{2}, \hat{x}_{3}, \hat{y}_{3}\right\}$ as functions of $\left\{\hat{x}_{1}, \hat{y}_{1}, \hat{y}_{2}\right\}$. Note that any solution to (6) is indeed a solution to (5) after a scaling of cable tension values. Further, we may compute the position of the robots $\tilde{q}_{i}$ given the normalized coordinates $\hat{q}_{i}$ and the kinematic constraint (4) as

$$
\tilde{q}_{i}=l_{i} \frac{\hat{q}_{i}}{\left\|\hat{q}_{i}\right\|}+P_{i} .
$$

By using $\hat{q}_{i}$ as coordinates, we can obtain closed-form analytic solutions for the positions of all robots that respect the kinematic constraints and the condition for static equilibrium.

If $\tilde{q}_{i}$ are chosen to satisfy the equations of equilibrium (3), the multipliers can be obtained by:

$$
\lambda=-\tilde{\mathbf{W}}^{\dagger} \tilde{\mathbf{g}},
$$

where $\tilde{\mathbf{W}}^{\dagger}$ is the Moore-Penrose inverse of $\tilde{\mathbf{W}}$. This allows us to check to see if the equilibrium pose yields tensions that satisfy (non-negative) lower and upper bounds. Figure 3 depicts a representation of the bounded and positive tension workspace for various configurations.

\section{B. The pose of the payload for hovering robots}

Problem 2 (Direct Problem). Given the actual positions of the robots, $\left\{q_{1}, q_{2}, q_{3}\right\}$, find the payload position(s) and orientation(s) satisfying the kinematics of the robots-cablespayload system (4) and the equations of equilibrium (3).

With hovering robots and cables in tension, we can treat the object as being attached to three stationary points through rigid rods and ball joints to arrive at the constraints in (4). Accordingly the object has three degrees of freedom. Imposing the three equilibrium conditions in (5) we can, in principle, determine a finite number of solutions for this analog of the direct kinematics problem. While this theoretical setting allows the analysis of the number of solutions using basic tools in algebraic geometry, very little can be established when the idealization of rigid rods is relaxed to include limits on cable tensions. Therefore we pursue a numerical approach to the 


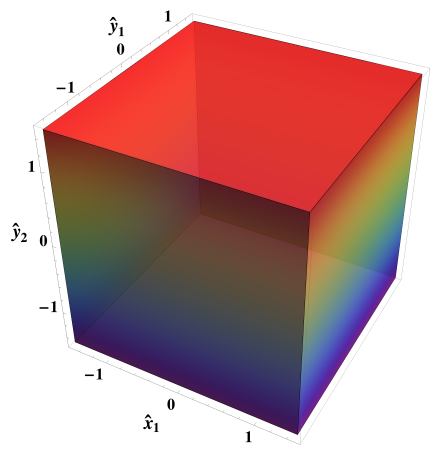

(a) $\alpha=\beta=0, \lambda>0$

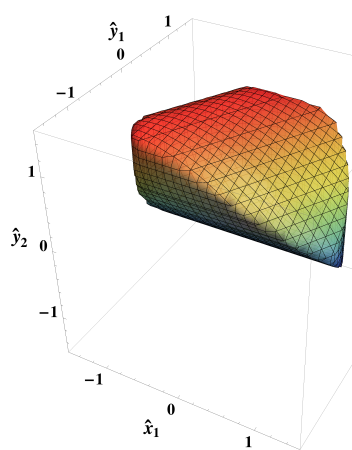

(c) $\alpha=0.3, \beta=0,0<\lambda<\frac{1}{2} m g$

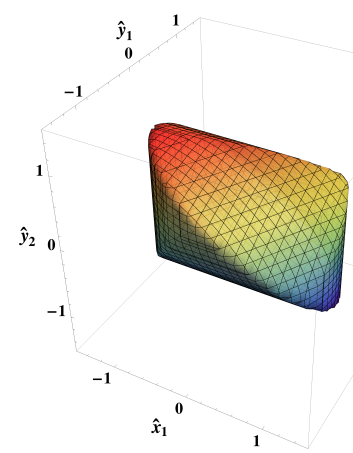

(b) $\alpha=\beta=0,0<\lambda<\frac{1}{2} m g$

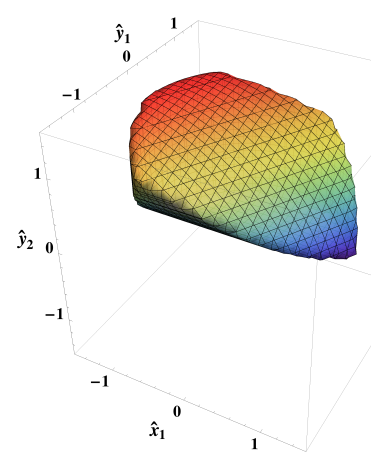

(d) $\alpha=\beta=0.3,0<\lambda<\frac{1}{2} m g$
Fig. 3. For a payload with mass $m=0.25 \mathrm{~kg}$ and $\tilde{x}_{2}^{p}=1 \mathrm{~m}, \tilde{x}_{3}^{p}=0.5 \mathrm{~m}$, and $\tilde{y}_{3}^{p}=0.87 \mathrm{~m}$, the numerically determined workspace of valid tensions in the normalized coordinates space $\left\{\hat{x}_{1}, \hat{y}_{1}, \hat{y}_{2}\right\}$. Any point selected in these valid regions meets the conditions of static equilibrium while ensuring positive and bounded tensions for all robots. Note that in these plots we do not consider inter-robot collisions (as compared to Fig. 9(a)).

\begin{tabular}{|c|c|c|c|}
\hline & $\lambda_{1}$ & $\lambda_{2}$ & $\lambda_{3}$ \\
\hline 1 & 0.06 & 0.80 & 5.48 \\
\hline 2 & -2.72 & 0.37 & 1.57 \\
\hline 3 & -3.11 & 0.03 & 2.56 \\
\hline 4 & -2.64 & 0.27 & 7.50 \\
\hline 5 & -5.54 & -1.19 & -0.61 \\
\hline
\end{tabular}

TABLE I

EIGENVALUES, $\lambda_{i}$, OF (9) DEFINED B Y THE EQUILIBRIUM CONFIGURATIONS FOR THE REPRESENTATIVE EXAMPLE (FIG. 4(B)).

problem by considering an alternative formulation. To numerically solve the direct problem, we formulate an optimization problem which seeks to minimize potential energy of the payload for a given robot configuration and payload geometry:

$$
\begin{aligned}
\underset{p_{i}}{\arg \min } & m g z_{C} \\
\text { s.t. } & \left\|p_{i}-q_{i}\right\| \leq l_{i}, i=\{1,2,3\} \\
& \left\|p_{i}-p_{j}\right\|=L_{i j}, i, j=\{1,2,3\}, i \neq j
\end{aligned}
$$

where the objective function is linear in terms of $p_{i}$ (assuming a planar payload), and $L_{i j}$ is the metric distance between two anchor points $p_{i}$ and $p_{j}$.

\section{Stability analysis}

Assuming that each robot, $q_{i}$, remains stationary, we wish to find the change in the potential energy of the payload. Define

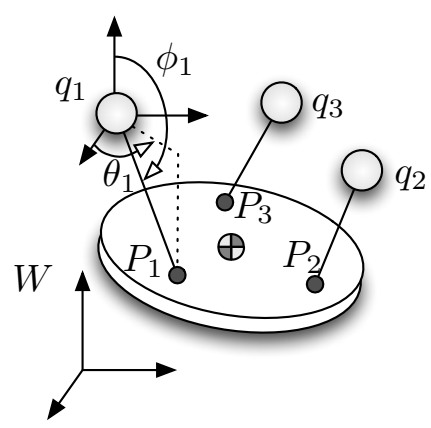

(a)

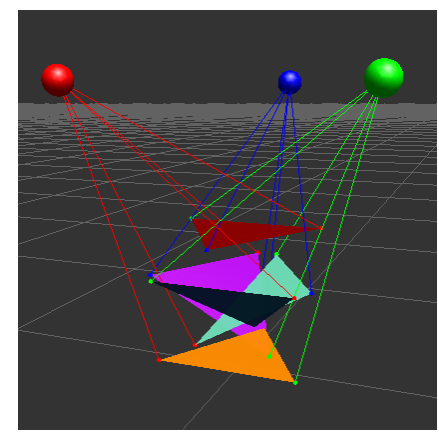

(b)
Fig. 4. Determining the pose of the payload for hovering robots via spherical coordinates (Fig. 4(a)). Given a representative robot configuration, five equilibrium solutions for the pose of the payload are found via the methods in Sect. III-B (Fig. 4(b)). The only stable configuration is orange (as noted in Table I).

the height of the payload $z_{C}$ as the center of mass, which we assume corresponds to the geometric center of the payload. Therefore,

$$
z_{C}=\frac{1}{3} \sum_{i=1}^{3}\left(z_{i}+l_{i} \cos \phi_{i}\right),
$$

where $\phi_{i}$ is defined in terms of spherical coordinates as depicted in Fig. 4(a). From (7), we may derive the potential energy of the payload as

$$
V=m g z_{C}=\frac{m g}{3} \sum_{i=1}^{3}\left(z_{i}+l_{i} \cos \phi_{i}\right),
$$

where $m$ is the mass of the payload and $g$ is acceleration due to gravity. To study the stability of the payload, we consider the second-order changes of the potential energy. We first note that the robot-cables-payload system has three degrees of freedom when requiring that cables be in tension. Therefore, we choose to parameterize the solution of the potential energy with respect to $\left\{\phi_{1}, \theta_{1}, \phi_{2}\right\}$ and define the Hessian of (8) as

$$
\mathcal{H}\left(\phi_{1}, \theta_{1}, \phi_{2}\right)=\left[\begin{array}{ccc}
\frac{\partial^{2} V}{\partial \phi_{1}^{2}} & \frac{\partial^{2} V}{\partial \phi_{1} \partial \theta_{1}} & \frac{\partial^{2} V}{\partial \phi_{1} \partial \phi_{2}} \\
\frac{\partial^{2} V}{\partial \theta_{1} \partial \phi_{1}} & \frac{\partial^{2} V}{\partial \theta_{1}^{2}} & \frac{\partial^{2} V}{\partial \theta_{1} \partial \phi_{2}} \\
\frac{\partial^{2} V}{\partial \phi_{2} \partial \phi_{1}} & \frac{\partial^{2} V}{\partial \phi_{2} \partial \theta_{1}} & \frac{\partial^{2} V}{\partial \phi_{2}^{2}}
\end{array}\right] .
$$

Due to space constraints, we defer the presentation of the analytic derivation of the entries of (9) to [10] where we show that a closed-form analytic representation of (9) is possible, enabling stability analysis of robots-cables-payload configurations. In Fig. 4(b), we provide the resulting set of equilibrium solutions to the direct problem for a representative example robot configuration and the corresponding numerical stability analysis in Table I.

\section{EXPERIMENTATION}

\section{A. The quadrotor robots}

In Sect. II, we assume point-model aerial robots but in Sect. V provide results on a team of commercially available quadrotors (see Fig. 5). In this section we develop a transformation from desired applied forces to control inputs required 


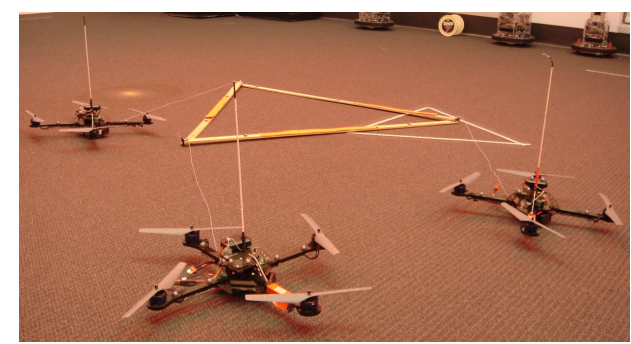

Fig. 5. The aerial robots and manipulation payload for experimentation. The payload is defined by $m=0.25 \mathrm{~kg}$ and $\tilde{x}_{2}^{p}=1 \mathrm{~m}, \tilde{x}_{3}^{p}=0.5 \mathrm{~m}$, and $\tilde{y}_{3}^{p}=0.87 \mathrm{~m}$, with $l_{i}=1 \mathrm{~m}$.

by the hardware platform. Some of this discussion is specific to the quadrotor used for our experiments.

1) Model: We begin by considering an aerial robot in $\mathbb{R}^{3}$ with mass $m$ and position and orientation, $q=[x, y, z] \in \mathbb{R}^{3}$, and $\mathbf{R}(\alpha, \beta, \gamma) \in S O(3)$, respectively. We do not have direct access to the six control inputs for linear and angular force control, but instead have access to four control inputs $\nu=$ $\left[\nu_{1}, \ldots, \nu_{4}\right]$ defined over the intervals $\left[\nu_{1}, \nu_{2}, \nu_{3}\right] \in[-1,1]$ for the orientations and $\nu_{4} \in[0,1]$ for the vertical thrust.

Based on system identification and hardware documentation, we arrive at the following dynamic model relating the control inputs and the forces and moments produced by the rotors in the body frame:

$$
\begin{aligned}
\tau_{x} & =-K_{v, x}^{q} \dot{\alpha}-K_{p, x}^{q}\left(\alpha-\nu_{1} \alpha_{\max }\right) \\
\tau_{y} & =-K_{v, y}^{q} \dot{\beta}-K_{p, y}^{q}\left(\beta-\nu_{2} \beta_{\max }\right) \\
\tau_{z} & =-K_{v, z}^{q} \dot{\gamma}-K_{p, z}^{q} \nu_{3} \gamma_{i n c} \\
f_{z} & =\nu_{4} f_{\max }
\end{aligned}
$$

where $\tau_{x}, \tau_{y}$, and $\tau_{z}$ are the torques along the body-fixed $x$, $y$, and $z$ axes, respectively, and $f_{z}$ is the force (thrust) along the body-fixed $z$ axis.

The relevant parameters above are defined as:

- $K_{p}^{q}, K_{v}^{q}$ - feedback gains applied by the aerial robot;

- $f_{\max }$ - maximum achievable thrust as a non-linear function of battery voltage;

- $\alpha_{\max }, \beta_{\max }$ - maximum achievable roll and pitch angles;

- $\gamma_{\text {inc }}$ - conversion parameter between the units of $\nu_{3}$ and radians.

All of these parameters were identified through system identification. Therefore, in the discussion that follows, we consider the following desired inputs:

$$
\left[\begin{array}{c}
\alpha^{d} \\
\beta^{d} \\
\gamma^{d} \\
f_{z}^{d}
\end{array}\right]=\left[\begin{array}{llll}
\nu_{1} & \nu_{2} & \nu_{3} & \nu_{4}
\end{array}\right]\left[\begin{array}{c}
\alpha_{\max } \\
\beta_{\max } \\
\gamma_{i n c} \\
f_{\max }
\end{array}\right],
$$

noting that we can compute the control inputs $\nu$ given the desired values assuming the parameters are known.

\section{B. Controllers}

We now derive a transformation from the control inputs for our point-model abstraction in the world frame to the control inputs $\nu$ in the body-frame described in Sect. IV-A. The dynamics of the point robot in the world frame can be written as:

$$
m I_{3} \ddot{q}=F_{g}+\left[\begin{array}{c}
F_{x} \\
F_{y} \\
F_{z}
\end{array}\right] .
$$

where $F_{x}, F_{y}$, and $F_{z}$ are the control input forces in the world frame and $F_{g}$ is the force due to gravity. These can be related to the forces in the robot body frame:

$$
\left[\begin{array}{c}
0 \\
0 \\
f_{z}
\end{array}\right]=\mathbf{R}(\alpha, \beta, \gamma)^{-1}\left[\begin{array}{c}
F_{x} \\
F_{y} \\
F_{z}
\end{array}\right] .
$$

Thus, for a given (current) $\gamma$, there is a direct relationship between the triples $\left(\alpha, \beta, f_{z}\right)$ and $\left(F_{x}, F_{y}, F_{z}\right)$. Solving (12) results in sixteen solutions for $\left(\alpha, \beta, f_{z}\right)$, eight of which require $f_{z} \leq 0$. Requiring that the robot always apply thrust with $F_{z}>0$ reduces the solution set to the following:

$$
\begin{aligned}
f_{z} & =\sqrt{F_{x}^{2}+F_{y}^{2}+F_{z}^{2}} \\
\alpha & = \pm \cos ^{-1}\left(\frac{\sqrt{\alpha_{n}}}{\sqrt{2} f_{z}}\right) \\
\alpha_{n} & =F_{x}^{2}+2 F_{x} F_{y} \sin (2 \gamma)+F_{y}^{2}+2 F_{z}^{2}+\left(F_{x}^{2}-F_{y}^{2}\right) \cos (2 \gamma) \\
\beta & = \pm \cos ^{-1}\left(\frac{F_{z}}{\sqrt{F_{z}^{2}+\left(F_{x} \cos (\gamma)+F_{y} \sin (\gamma)\right)^{2}}}\right) .
\end{aligned}
$$

From (13), it is clear that two solution sets are valid, but only for specific intervals. For example, consider pitch $(\beta)$ when applying controls in the yaw directions $[-\pi / 2,0, \pi / 2, \pi]$ with $\left\{F_{x}, F_{y}, F_{z}\right\}>0$. For each of the four cases respectively, we expect $\beta<0, \beta>0, \beta>0$, and $\beta<0$. A similar argument may be made for $\alpha$. Therefore, we must construct a piecewise-smooth curve as a function of the external forces $\left\{F_{x}, F_{y}\right\}$ and $\gamma$ over the intervals $\left[-\pi, \gamma_{1}\right],\left[\gamma_{1}, \gamma_{2}\right],\left[\gamma_{2}, \pi\right]$, defined by the zero points of the conditions in (13). For $\alpha$, we find that

$$
\begin{aligned}
{\left[\gamma_{\alpha_{1}}, \gamma_{\alpha_{2}}\right] } & =-\tan ^{-1}\left(\frac{F_{x}}{F_{y}}\right) \\
& +\left\{\begin{array}{cc}
{\left[-\frac{3 \pi}{2},-\frac{\pi}{2}\right]} & \text { if }-\tan ^{-1}\left(\frac{F_{x}}{F_{y}}\right)>\frac{\pi}{2} \\
{\left[-\frac{\pi}{2}, \frac{\pi}{2}\right]} & \text { otherwise }
\end{array}\right.
\end{aligned}
$$

In a similar manner, we find for $\beta$

$$
\begin{aligned}
{\left[\gamma_{\beta_{1}}, \gamma_{\beta_{2}}\right] } & =2 \tan ^{-1}\left(\sqrt{1+\frac{F_{x}^{2}}{F_{y}^{2}}}-\frac{F_{x}}{F_{y}}\right) \\
& +\left\{\begin{array}{cc}
{\left[-\frac{3 \pi}{2},-\frac{\pi}{2}\right]} & \text { if } \tan ^{-1}\left(\sqrt{1+\frac{F_{x}^{2}}{F_{y}^{2}}}-\frac{F_{x}}{F_{y}}\right)>\frac{\pi}{4} \\
{\left[-\frac{\pi}{2}, \frac{\pi}{2}\right]} & \text { otherwise }
\end{array}\right.
\end{aligned}
$$

We now construct piecewise-smooth solutions that reflect the expected inputs for all values of $\gamma$. From (13), we denote the positive $\alpha$ solution as $\alpha_{+}$and the negative solution as $\alpha_{-}$. The piecewise-smooth input for $\alpha$ is

$$
\alpha= \begin{cases}\left\{\alpha_{+}, \alpha_{-}, \alpha_{+}\right\} & \text {if } F_{y}>0 \\ \left\{\alpha_{-}, \alpha_{+}, \alpha_{-}\right\} & \text {otherwise }\end{cases}
$$


In a similar manner, for $\beta$ we find

$$
\beta= \begin{cases}\left\{\beta_{-}, \beta_{+}, \beta_{-}\right\} & \text {if } F_{x}>0 \\ \left\{\beta_{+}, \beta_{-}, \beta_{+}\right\} & \text {otherwise }\end{cases}
$$

Both $\alpha$ and $\beta$ become singular when $F_{y}=0$. However, it is clear from the definitions in (13) that at this singularity for the intervals $[-\pi, 0]$ and $[0, \pi]$,

$$
\alpha= \begin{cases}\left\{\alpha_{-}, \alpha_{+}\right\} & \text {if } F_{x}>0 \\ \left\{\alpha_{+}, \alpha_{-}\right\} & \text {otherwise }\end{cases}
$$

and for the intervals $[-\pi,-\pi / 2],[-\pi / 2, \pi / 2]$, and $[\pi / 2, \pi]$

$$
\beta= \begin{cases}\left\{\beta_{-}, \beta_{+}, \beta_{-}\right\} & \text {if } F_{x}>0 \\ \left\{\beta_{+}, \beta_{-}, \beta_{+}\right\} & \text {otherwise }\end{cases}
$$

Of course, there remains the trivial solution of $\alpha=\beta=0$ when $F_{x}=F_{y}=0$.

Therefore, we may define a force $F=\left[F_{x}, F_{y}, F_{z}\right]^{\mathrm{T}}$ in the world frame that is transformed into appropriate control inputs via $(10,12)$. To this end, we compute $F$ in implementation based on proportional-integral-derivative (PID) feedback control laws determined by the desired robot configurations with feedforward compensation based on (11). For the purposes of this work, we additionally control $\nu_{3}$ to drive $\gamma$ to zero.

As a limitation of this approach, we note that the controller above results in thrust $f_{z}$ for any desired input. Therefore, when the robot is hovering at a level pose $(\alpha=\beta=0)$, a desired horizontal motion for which $F_{x}$ must be nonzero and $F_{z}=m g$, results in an unintended vertical thrust $f_{z}>m g$ until the robot rotates from the level pose.

\section{Payload model}

The payload is a rigid frame with cable attachment points given by $\tilde{x}_{2}^{p}=1 \mathrm{~m}, \tilde{x}_{3}^{p}=0.5 \mathrm{~m}$, and $\tilde{y}_{3}^{p}=0.87 \mathrm{~m}$ with mass $m=0.25 \mathrm{~kg}$. The cable lengths are equal with $l_{i}=1 \mathrm{~m}$.

\section{Simulation and Experiment Design}

Our software and algorithms are developed in $\mathrm{C} / \mathrm{C}++$ using Player/Gazebo [11] and interfaced with MATLAB for high-level configuration specification for both simulation and experiments.

Experiments are conducted with the AscTec Hummingbird quadrotor [12] from Ascending Technologies $\mathrm{GmbH}$ with localization information being provided by a Vicon motion capture system [13] running at $100 \mathrm{~Hz}$ with millimeter accuracy. Control commands are sent to each robot via Zigbee at $20 \mathrm{~Hz}$. The quadrotor is specified to have a payload capacity of $0.2 \mathrm{~kg}$ with a physical dimension conservatively bound by a sphere of radius $R=0.3 \mathrm{~m}$.

Robots can be positioned inside the $6.7 \mathrm{~m} \times 4.4 \mathrm{~m} \times 2.75 \mathrm{~m}$ workspace with an error bound of approximately $\pm 0.05 \mathrm{~m}$ in each direction. However, as the robots experience the effects of unmodelled external loads and interactions during manipulation, errors increase to approximately $\pm 0.15 \mathrm{~m}$.

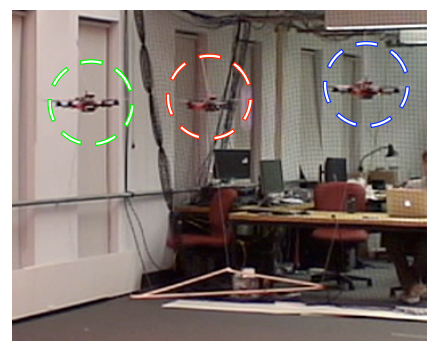

(a)

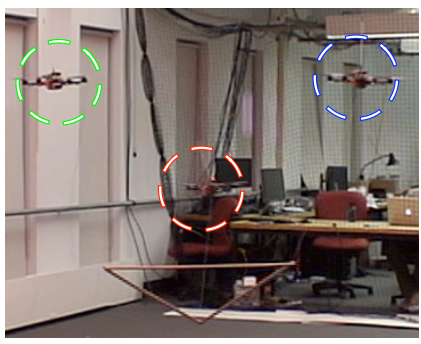

(b)
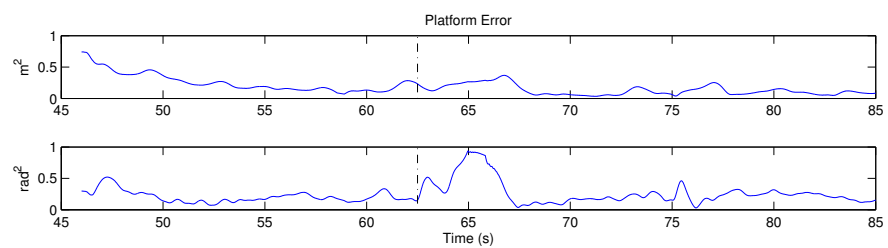

(c)

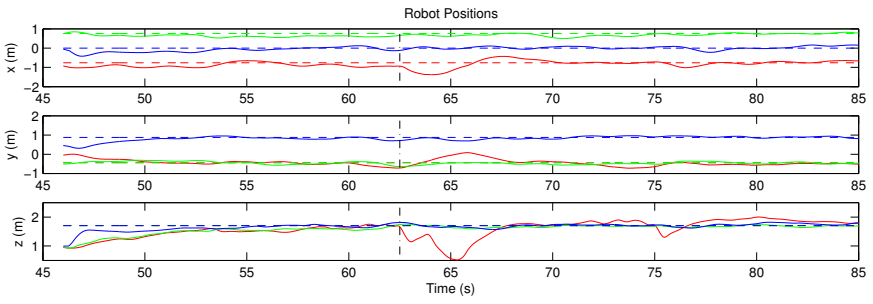

(d)

Fig. 6. In Fig. 6(a) the robots assume the desired configuration while in Fig. 6(b) one robot has suffered a large actuation failure. Figure 6(c) depicts the mean squared position and orientation error of the payload while Fig. 6(d) provides data on the individual robot control error. Vertical dashed lines in Figs. 6(c) and 6(d) show the exact time of the robot actuator failure.

\section{Results}

Using the solution to the inverse problem presented in Sect. III and the abstraction of a fully dynamic aerial vehicle to a point robot in Sect. IV, we are able to experimentally verify our ability to lift, transport, and manipulate a six degree of freedom payload by controlling multiple quadrotors.

\section{A. Cooperative Lifting}

We start with a symmetric configuration similar to that in Fig. 4(b) for benchmarking the performance of the system. Figure 6(a) depicts the team of robots in this configuration raising the payload to a specified pose in the workspace. During the course of experimentation in this configuration, in one of the trials, a robot suffers a momentary actuator failure causing it to lose power and drop (see Fig. 6(b)). We use the data from this trial to demonstrate the team's ability to quickly recover and return to the desired configuration. Analysis of the data in Figs. 6(c) and 6(d) suggests that the time constant for the closed loop response of the system is around $1-1.5 \mathrm{~s}$.

\section{B. Cooperative manipulation and transport}

In this experiment, the system is tasked with manipulating the payload through a sequence of poses. For each pose, we compute the desired configuration $\left(q_{1}, q_{2}, q_{3}\right)$ for the robots 


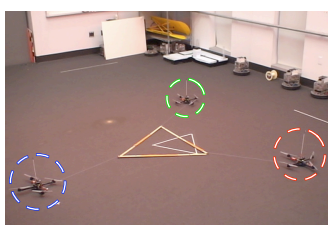

(a)

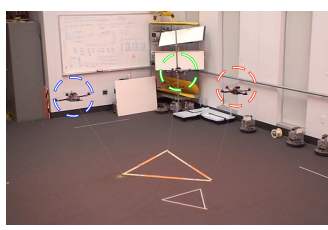

(b)

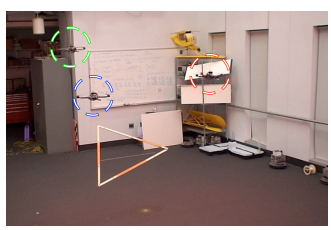

(c)

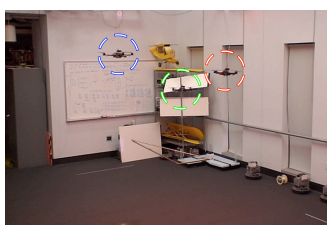

(d)

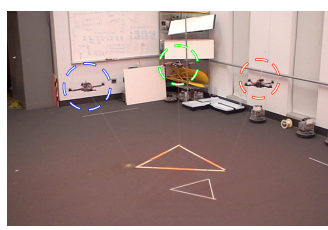

(e)

Fig. 7. Snapshots demonstrating cooperative manipulation and transportation. The team starts from initial conditions before takeoff (Fig. 7(a)), stabilizes the platform at each desired pose (Figs.7(b) - 7(d)), and returns the payload to the first pose before landing (Fig. 7(e)). Colored circles highlight individual robot positions during the evolution of the experiment. Videos of the experiments are available at http://kumar.cis.upenn.edu/movies/RSS2009.flv

and drive each robot to the desired goal. The underlying control system avoids inter-robot collisions via a simple potential field controller derived from the point-robot model in (11) with each robot modeled as a sphere of radius $R$, guaranteeing $\left\|q_{i}-q_{j}\right\|>2 R$ for all pairs of robots. This results in a smooth, though unplanned, trajectory for the payload. Figures 7 and 8 depict several snapshots during this experiment and the resulting system performance, respectively.

This experiment demonstrates that even though the manipulation system is underactuated for $n=3$, it is able to position and orient the payload as commanded. The small oscillations around each equilibrium configuration are inevitable because of the low damping and perturbations in the robot positions.

\section{Vi. Planning Multi-Robot MANIPULATION AND TRANSPORTATION TASKS}

In this section, we address motion planning for the aerial manipulation tasks and the generation of trajectories for the robots that respect (a) the kinematic workspace constraints; (b) the conditions of stable equilibrium for the payload; (c) constraints on the cable tensions $\left(\lambda_{\max } \geq \lambda_{i}>0\right)$; and (d) the geometric constraints necessary to avoid collisions $\left(\left\|q_{i}-q_{j}\right\|>2 R\right)$. In Sect. III, we computed $Q_{c}$ to to be the set of robot positions satisfying $(a, b)$ with positive tension in each cable. We now derive the effective workspace for the robots $Q_{M} \subset Q_{c}$ consisting of robot positions that satisfy (a-d) above. The planned trajectories of the robots must stay within $Q_{M}$ which has a complex shape owing to the nonsmooth constraints in (a-d).

Figure 9 illustrates the effective workspace $Q_{M}$ parameterized by $\left(\hat{x}_{1}, \hat{y}_{1}, \hat{y}_{2}\right)$. Indeed for a given payload pose, there are multiple points in the workspace that satisfy the conditions of stable equilibrium. Three representative conditions are shown in the figure. Thus during motion planning it is possible to optimize any number of design goals, including equal sharing of loads by the cooperating robots, robustness to disturbances to the payload, and maximizing the stability of the robotspayload system. To demonstrate this, we consider the response of the payload to an external disturbance in experimentation given two distinct robot configurations selected from the space of valid solutions $Q_{M}$ depicted in Fig. 9(a). The first mirrors the simulation configuration shown in Fig. 9(b) while the second configuration is selected to maximize the smallest natural frequency of the payload given by the smallest eigenvalue of the Hessian in (9). Figure 10 shows that the robot configuration

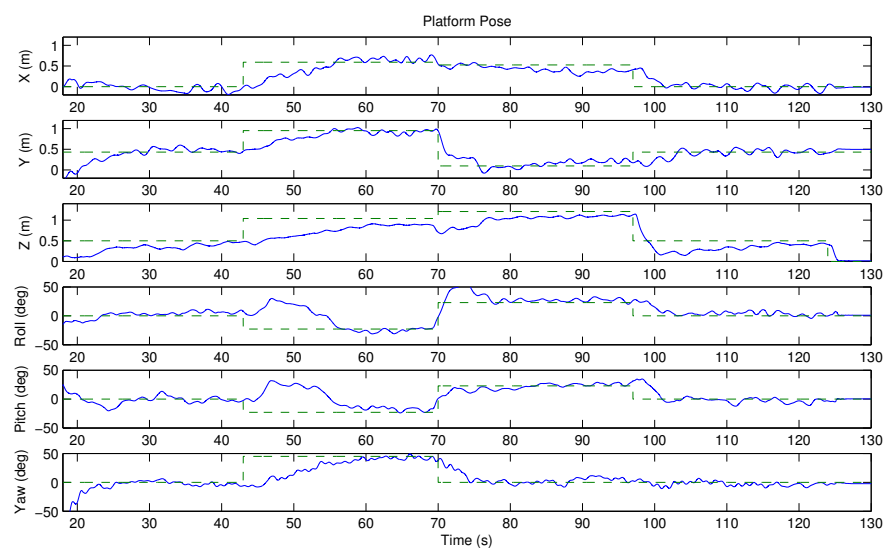

(a)
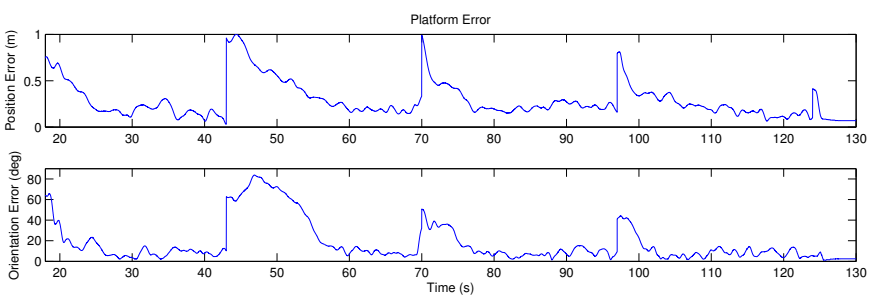

(b)

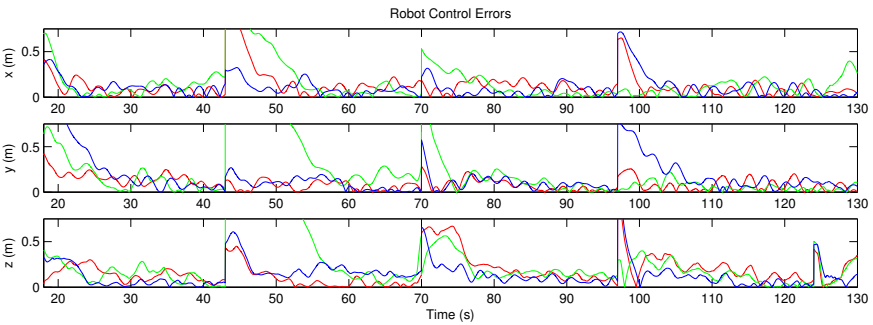

(c)

Fig. 8. Data from the manipulation and transportation experiment, including: pose data for the payload overlaid with a dashed line representing desired values (Fig. 8(a)), aggregate position and orientation error of the payload (Fig. 8(b)), and individual robot control errors (Fig. 8(c)).

determined by the natural frequency-based measure attenuates the payload error more quickly than the other configuration in which the lower natural frequency results in oscillations that take longer to damp out.

In future work we will further investigate the planning of aerial manipulation by exploring the use of sample-based planning methods for payload trajectory control. While the 


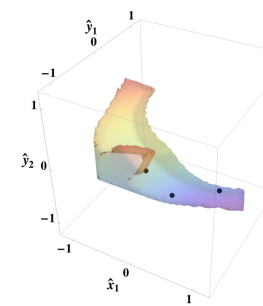

(a)

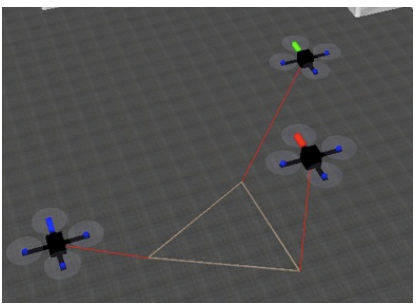

(c)

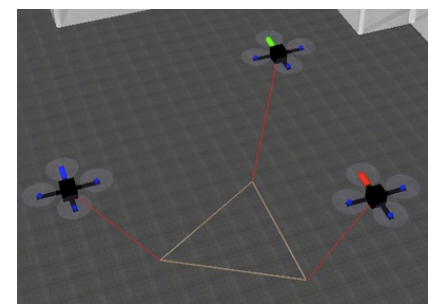

(b)

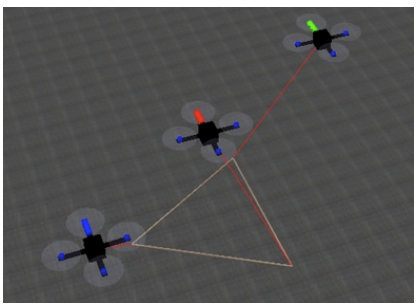

(d)
Fig. 9. Various points in $Q_{M}$ for $\alpha=\beta=0$ (Figs. 9(b)-9(d)). Figure 9(a) depicts numerically determined regions of valid tensions in the space of $\hat{q}$ requiring $\lambda_{i}<\frac{1}{2} m g$ and $\left\|q_{i}-q_{j}\right\|>1 \mathrm{~m}$ (for collision avoidance), with black points indicating the configurations selected in Figs. 9(b)-9(d). For completeness, the normalized coordinates, $\left\{\hat{x}_{1}, \hat{y}_{1}, \hat{y}_{2}\right\}$, of each configuration follows: $\{-0.2724,-0.3054,-0.3054\}$ (Fig. 9(b)), $\{0,0,-0.9\}$ (Fig. 9(c)), $\{0.6,0.45,-0.9\}$ (Fig. 9(d)).

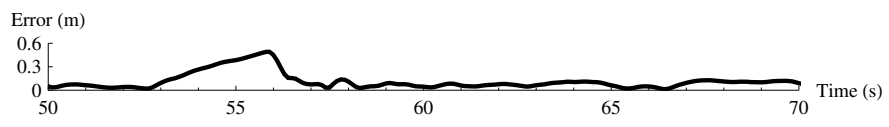

(a)

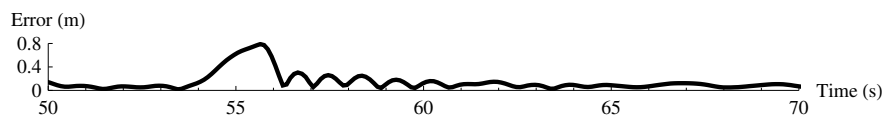

(b)

Fig. 10. A disturbance is applied to the payload in experimentation at time $55 \mathrm{~s}$ in two trials. In Fig. 10(a), the robot configuration is selected based on the maximization of the natural frequency of the payload and in Fig. 10(b), the robot configuration is chosen to be similar to that shown in Fig. 9(b). Note that the configuration in Fig. 10(a) attenuates error more quickly.

analytic representation of our feasible workspace is complicated, fast numerical verification suggests standard samplebased methods can be used for this task. Additionally, while it is possible that the workspace of configurations with tension limits is not simply connected, in our extensive numerical studies, we have not found this to happen with realistic values of geometric parameters and tension bounds. This suggests that it should be possible to transition smoothly from one configuration to another without loss of cable tension. Finally, the fact that the direct problem has multiple stable solutions is a potential source of concern in real experimentation since positioning the robots at a desired set of positions does not guarantee that the payload is at the desired position and orientation. In a forthcoming paper [14], we show that by further constraining $Q_{M}$, we can reduce the direct problem to a Second Order Cone Program (SOCP). We plan to incorporate these constraints into our motion planning algorithm.

\section{CONCLUSION AND Future Work}

We presented a novel approach to aerial manipulation and transport using multiple aerial robots. We derived a mathematical model that captures the kinematic constraints and the mechanics underlying stable equilibria of the underactuated system. The number of unconstrained degrees of freedom is equal to six less the number of robots. We also presented an experimental implementation and results that suggest that cooperative manipulation can be used as an effective way of manipulating and transporting payloads that are beyond the capability of individual micro UAVs.

The main limitation of our approach lies in our inability to damp out oscillations in the underdamped system. Because of this, our trajectory following capabilities are limited to slow motions with harmonics well under the fundamental frequencies of around $3-5 \mathrm{~Hz}$. One possibility is to use the robots to actively damp out oscillations using methods analogous to controlling flexible manipulators. We are currently engaged in a more thorough study of the underlying joint configuration space and the effects of cable constraints with a view to developing motion planning algorithms. We are also considering the application of control and estimation methods that relax our current reliance on globally available state information and enable a better understanding of the effects of sensing and actuation uncertainty on control performance.

\section{REFERENCES}

[1] R. M. Murray, "Trajectory generation for a towed cable system using differential flatness," in IFAC World Congress, San Francisco, CA, July 1996.

[2] P. Cheng, J. Fink, S. Kim, and V. Kumar, "Cooperative towing with multiple robots," in Proc. of the Int. Workshop on the Algorithmic Foundations of Robotics, Guanajuato, Mexico, Dec. 2008.

[3] E. Stump and V. Kumar, "Workspaces of cable-actuated parallel manipulators," ASME Journal of Mechanical Design, vol. 128, no. 1, pp. 159-167, Jan. 2006.

[4] R. Verhoeven, "Analysis of the workspace of tendon-based stewart platforms," Ph.D. dissertation, University Duisburg-Essen, Essen, Germany, July 2004

[5] S. R. Oh and S. K. Agrawal, "A control lyapunov approach for feedback control of cable-suspended robots," in Proc. of the IEEE Int. Conf. on Robotics and Automation, Rome, Italy, Apr. 2007, pp. 4544-4549.

[6] P. Bosscher and I. Ebert-Uphoff, "Wrench-based analysis of cable-driven robots," in Proc. of the IEEE Int. Conf. on Robotics and Automation, vol. 5, New Orleans, LA, Apr. 2004, pp. 4950-4955.

[7] K. H. Hunt, Kinematic Geometry of Mechanisms. Oxford University Press, 1978.

[8] J. Phillips, Freedom in Machinery. Cambridge University Press, 1990, vol. 1.

[9] J. M. Selig, Geometric Fundamentals of Robotics. Springer, 2005.

[10] N. Michael, S. Kim, J. Fink, and V. Kumar, "Kinematics and statics of cooperative multi-robot aerial manipulation with cables," University of Pennsylvania, Philadelphia, PA, Tech. Rep., Mar. 2009, Available online at http://www.seas.upenn.edu/ nmichael.

[11] B. P. Gerkey, R. T. Vaughan, and A. Howard, "The Player/Stage Project: Tools for multi-robot and distributed sensor systems," in Proc. of the Int. Conf. on Advanced Robotics, Coimbra, Portugal, June 2003, pp. 317323.

[12] “Ascending Technologies, GmbH," http://www.asctec.de.

[13] "Vicon Motion Systems, Inc." http://www.vicon.com.

[14] J. Fink, N. Michael, S. Kim, and V. Kumar, "Planning and control for cooperative manipulation and transportation with aerial robots," in Int. Symposium of Robotics Research, Zurich, Switzerland, Aug. 2009, To Appear. 